\title{
Programas educativos musicales en la educación no formal para la atención a jóvenes en vulnerabilidad socioeconómica. Configuración de una red de traducciones e intereses
}

Musical education programs of informal education that look after socioeconomically vulnerable teenagers. Configuration of a network of translations and interests

Elsa Natali González González Lcengg@gmail.com

Departamento de Psicología y Ciencias de la Comunicación Universidad de Sonora

Sonora, México

ORCID: http://orcid.org/0000-0003-4008-6948

Leonel De Gunther Delgado

lgunthere@capomo.uson.mx

Departamento de Bellas Artes

Universidad de Sonora

Sonora, México

ORCID: http://orcid.org/0000-0002-8075-0818

doi: 10.7203/LEEME.46.16310

Recibido: 30-11-2019 Aceptado: 29-06-2020. Contacto y correspondencia: Elsa Natali González González. Departamento de Psicología y Ciencias de la Comunicación, Universidad de Sonora, Boulevar Luis Encinas y Rosales, Colonia centro, Hermosillo, s/n, C.P. 83000 Sonora. México.

\section{Resumen}

El análisis en red de un programa no formal de educación a través de las artes, que atiende a jóvenes en vulnerabilidad socioeconómica, permite reconocer sus relaciones, alcances y límites en otras redes (el programa, lo familiar, lo educativo formal y los lazos entre amigos). De ahí que el objetivo de este trabajo se centre en construir la red de asociaciones entre dos de estas redes: el programa y los lazos entre amigos ${ }^{1}$. En concordancia con este objetivo, adecuamos la teoría del actor-red (TAR) y empleamos recursos etnográficos para la recolección y análisis de los datos. Entre los principales hallazgos destacamos que las controversias funcionan como el disparador que genera movimientos de configuración, estabilización o desvanecimiento de las redes. Asimismo, el ensamblaje de la red describe los procesos que están detrás de las aportaciones o cambios que le son atribuidos a la educación a través de las artes.

Palabras clave: Artes; música; educación a través de las artes; educación no formal.

Abstract

The network analysis of a non-formal program of education through the arts, which look after socioeconomically vulnerable teenagers, allows us to recognize their relationships, scope, and limits in other networks (the program, the family, the formal education, and the linkage between friends). The objective of this work is focused on building the network of associations between two of these networks: the program and the linkages between friends. In accordance with this objective, we adapted the actornetwork theory (ANT) and we used ethnographic resources for data collection and analysis. Among the main findings, we highlight that controversies work as the trigger which generates in networks configuration movements, stabilization, or their fading. Likewise, the network assembly describes the processes that are behind the contributions or changes which are attributed to education through the arts.

Key words: Art; music; education through arts; non-formal education.

\footnotetext{
${ }^{1}$ Este estudio es parte de una investigación más amplia. Sin embargo, debido a la amplitud del de la red se centra la atención solo en el vínculo entre el programa y los lazos entre amigos.

@Elsa Natali González González y Leonel De Gunther Delgado. The content of this article is the sole responsibility of the authors. The Revista Electrónica de LEEME and Universitat de València are not liable for any legal actions that may arise involving the article's content. Revista Electrónica de LEEME - Lista Electrónica Europea de Música en la Educación-. http://ojs.uv.es/index/php/LEEME/index ISSN: 1575-9563. Editores: Universidad de Valencia y Jesús Tejada. Visibilidad de esta revista: SCOPUS, Emerging Sources Citation Index (Clarivate), EBSCO, CINDOC (CSIC), Citefactor, COPAC, Dialnet, DICE (CSIC), DOAJ, e-revistas (CSIC), EBSCO Premier, ERIH+, Gale Cengage Learning, IN-RECS, IRESIE, LATINDEX, MIAR, OCLC Worldcat, RESH, REDIB, RILM Core Journals, SUDOC, ULRICHS. Esta revista es de acceso libre mediante licencia Creative Commons 4.0 CC by. Política de archivado: etiqueta verde SHERPA-ROMEO.
} 


\section{Introducción}

Tod Machover, profesor del Media Lab del Massachusetts Institute of Technology, invita a reconocer que el violonchelo como objeto musical no discreto o limitado es parecido a lo humano. Tiene su tamaño, tiene el sonido de su voz, se acopla al cuerpo, se adapta casi siempre a las situaciones de la vida cotidiana. Es un compañero para pensar, para descubrir. Es susceptible de transformarse en otro instrumento; medio de convivencia; de articulación entre pensamiento y práctica; de extensión hacia otras redes (Machover, 2007).

Para Latour (2010), lo expresado en el párrafo anterior trata de una composición que escapa del recorte de la ciencia actual, lo cual permite una comprensión articulada de un acontecimiento en red. De la misma manera, cuando Michel Cassé, astrofísico francés elabora su respuesta a la pregunta “¿Qué ve un astrofísico en una copa de vino de Burdeos?" muestra la paradójica constitución humana. Estamos constituidos por objetos físicos, químicos y vivos interconectados en nuestro interior en una compleja red que produce la vida, pero somos capaces de abstraerlos a través de nuestro pensamiento y de diferentes dominios de conocimiento científico. De ahí que existe una "doble condición humana, una natural y otra metanatural" (Morin, 2002, p.39). Sin embargo, objeto y acontecimiento son aquí por un lado discretos, con límites reconocibles y, por el otro, no discretos (Silva, 2011). En este último caso, constituyen una densa red de traducciones que se asocian a otras redes.

En este sentido, el violonchelo se articula con otros objetos y acontecimientos en una compleja red de asociaciones, cuyas posibles combinaciones crean nuevas redes. El violonchelo se convierte en un objeto no discreto que se traduce mientras viaja por una densa red de dominios como la ciencia, el arte y la cultura, creando intereses en su articulación. Dominios que no son aislados ni discretos, sino brazos de un mismo organismo que se han desarrollado juntos. Las trayectorias de estos brazos se han cruzado de manera permanente en la historia, elaborando composiciones y pasajes distintos en la construcción del conocimiento humano (Latour, 2013).

Sherry Turkle suele hablar de esta composición a partir de objetos evocadores desde las nociones del bricolaje de Levi Strauss combinadas con las de Piaget de aprendizaje situado y los trabajos de Latour sobre objetos socio-técnicos como "una manera de combinar y recombinar un conjunto de materiales hasta construir nuevas ideas..."(Turkle, 2007, p.4). De ahí que, como señala Piaget (1975), nuestro aprendizaje es situado, lo que permite que a través de los objetos podemos pensar en números, espacios, tiempo, causalidad, etc., pasando nuestra vida inventándola y reinventándola. Un objeto como la capa de ozono, por ejemplo, interconecta ciencia, naturaleza, tecnología y política; en todo caso, ponen de manifiesto las relaciones sociales de nuestro tiempo.

@Elsa Natali González González y Leonel De Gunther Delgado. The content of this article is the sole responsibility of the authors. The Revista Electrónica de LEEME and Universitat de València are not liable for any legal actions that may arise involving the article's content. Revista Electrónica de LEEME - Lista Electrónica Europea de Música en la Educación-. http://ojs.uv.es/index/php/LEEME/index ISSN: 1575-9563. Editores: Universidad de Valencia y Jesús Tejada. Visibilidad de esta revista: SCOPUS, Emerging Sources Citation Index (Clarivate), EBSCO, CINDOC (CSIC), Citefactor, COPAC, Dialnet, DICE (CSIC), DOAJ, e-revistas (CSIC), EBSCO Premier, ERIH+, Gale Cengage Learning, IN-RECS, IRESIE, LATINDEX, MIAR, OCLC Worldcat, RESH, REDIB, RILM Core Journals, SUDOC, ULRICHS. Esta revista es de acceso libre mediante licencia Creative Commons 4.0 CC by. Política de archivado: etiqueta verde SHERPA-ROMEO. 


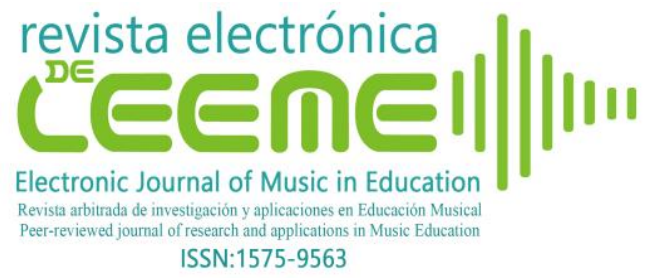

Así, los programas no formales de educación a través de las artes se dirigen más al desarrollo humano integral y la transformación sociocultural de las personas que a la formación artística profesional (Christophersen, 2015). En este sentido, comparten lógicas similares a los ejemplos anteriores, articulándose como una red o nodos de una red más amplia (Latour, 2013), en la que transita no sólo música, sino vínculos que van de los programas a través de las artes a la familia, a la escuela y a los lazos entre amigos multidireccionalmente, lo que permite una comprensión más amplia del fenómeno y de su configuración. Sin embargo, dada la complejidad de la red, según Latour (2008), deben tratarse a detalle, por lo que el presente análisis se centra en el programa y su asociación con la red de lazos entre amigos, debido a la capacidad de esta última para extenderse a otras redes.

De lo anterior se desprenden dos preguntas de investigación ¿cómo se configura la red de Hermosiarte, en particular entre el programa y los lazos entre amigos? Y, con ello, ¿cuáles son las implicaciones de la educación a través de las artes? Para ello planteamos la teoría del actor-red para atender tal problemática.

Desde esta lógica el objetivo consiste en construir la red de asociaciones vinculada a los lazos entre amigos en un programa no formal de educación a través de las artes en el estado de Sonora, México. Constituye una manera de pensar relacionalmente un objeto de investigación, donde su existencia está dada a partir de sus relaciones con otros objetos. Tal objetivo responde a la complejidad de ensamblar lo social, aquello que transita en forma de redes, a veces invisibles, que redefinen el sentido social dentro de las prácticas producidas en los programas no formales de educación a través de las artes. Ello implica el empleo de otros soportes teóricos tales como la teoría del actor-red (TAR) de Latour (2008), la cual permite observar los alcances y limitaciones de este tipo de educación.

El trabajo se divide en cuatro partes, en la primera presentamos el problema de investigación y damos cuenta del orientador teórico; en la segunda, el esquema metodológico, en la tercera, el análisis de los resultados obtenidos y en la cuarta, las principales conclusiones de la investigación y su discusión.

\subsection{Del imaginario a la realidad: problemática de estudio}

Múltiples son los condicionamientos de la educación a través de las artes en su modalidad no formal. Si bien las aportaciones de las artes en la educación son reconocidas política, social y científicamente (CONEVAL y UNICEF, 2015), existe la necesidad de estudiar sus procesos como articuladores de estas. ¿Qué hace la educación a través de las artes como programa educativo no formal? Es una pregunta que se intenta responder en las investigaciones revisadas, sin alcanzar un logro específico.

@Elsa Natali González González y Leonel De Gunther Delgado. The content of this article is the sole responsibility of the authors. The Revista Electrónica de LEEME and Universitat de València are not liable for any legal actions that may arise involving the article's content. Revista Electrónica de LEEME - Lista Electrónica Europea de Música en la Educación-. http://ojs.uv.es/index/php/LEEME/index ISSN: 1575-9563. Editores: Universidad de Valencia y Jesús Tejada. Visibilidad de esta revista: SCOPUS, Emerging Sources Citation Index (Clarivate), EBSCO, CINDOC (CSIC), Citefactor, COPAC, Dialnet, DICE (CSIC), DOAJ, e-revistas (CSIC), EBSCO Premier, ERIH+, Gale Cengage Learning, IN-RECS, IRESIE, LATINDEX, MIAR, OCLC Worldcat, RESH, REDIB, RILM Core Journals, SUDOC, ULRICHS. Esta revista es de acceso libre mediante licencia Creative Commons 4.0 CC by. Política de archivado: etiqueta verde SHERPA-ROMEO. 


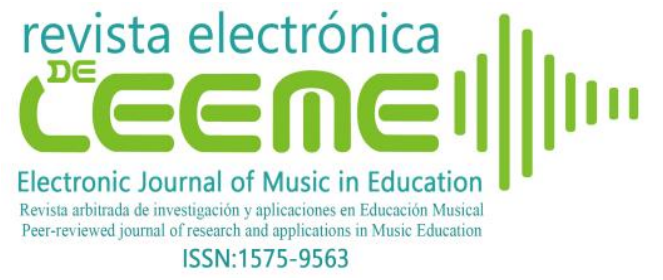

Esta pregunta se relaciona con el desarrollo de tales programas los cuales superan el análisis sistemático de sus aportaciones, sea por (1) la incomprensión de sus contribuciones en la práctica real (Rusinek, 2008), (2) por su poco valor frente a otras disciplinas (Ángel-Alvarado y Lira-Cerda, 2017), (3) por su reticencia a la evaluación, sea porque se evalúan y mejoran a sí mismos como modelos autorreferenciales, porque responden a intereses de quienes los financian y ocultan sus resultados, (4) porque los artistas se resisten a la evaluación, considerando que minimiza o diluye los beneficios reales de este tipo de programas (Berrón, 2017).

Por otro lado, existen estudios altamente sistematizados teórica y metodológicamente donde las artes son objeto de otras disciplinas, sin embargo, solo atienden a un fragmento de la realidad, dejando de lado otras relaciones, cuya omisión limita el reconocimiento de los alcances de la educación a través de las artes, en tanto no proveen información necesaria para reconocer las asociaciones vinculadas al programa ni de lo que transita ni lo que producen en red. Por lo que nos preguntamos, ¿cómo se configura la red de Hermosiarte, en particular entre el programa y los lazos entre amigos? Y, con ello, ¿cuáles son las implicaciones de la educación a través de las artes? Por ello planteamos la teoría del actor-red para atender tal problemática.

\subsection{La teoría del actor-red (TAR) en acción}

La TAR o ANT, por sus siglas en inglés, es un planteamiento que emerge en los años ochenta dentro de la sociología y se extiende a otros campos de conocimiento. En la educación se introduce a finales de los años noventa, amplificándose a partir del año dos mil (Fenwick y Edwards, 2012). Fundada por Callon, Law y Latour es conocida como sociología de las asociaciones, de las traducciones o mediaciones (Hennion, 2017). Concibe la sociedad como un tipo de conexión entre cosas, más que un sustrato que sirve como explicación de las cosas. Su posicionamiento es controversial frente a otras formas de comprender o explicar lo social, ya que lo social es aquí un movimiento muy peculiar de re-asociación y reensamblaje a través de agencias (Latour, 2008). Lo social, es entonces una red compuesta de centros de traducción en constante movimiento o configuración. De ahí que estos planteamientos indiquen que se trata de un campo no unificado de estudio o de una teoría no sistematizada.

Se comprende también como una etiqueta bajo la cual se designa un conjunto de principios de orden metodológico, epistémico y trabajos de campo en cuyo despliegue revolucionan la tradición del pensamiento social (Tirado y Domènech, 2005). De ahí que en educación se encuentren estudios que siguen procedimientos dados: problematizar, crear interesamientos, asociarse y movilizarse (Callon, Lawand y Rip, 1998/1986); o, tiendan a actualizar, reinterpretar o reintroducir otros aspectos teóricos en el uso de la TAR (Fenwick y Edwards, 2010).

@Elsa Natali González González y Leonel De Gunther Delgado. The content of this article is the sole responsibility of the authors. The Revista Electrónica de LEEME and Universitat de València are not liable for any legal actions that may arise involving the article's content. Revista Electrónica de LEEME - Lista Electrónica Europea de Música en la Educación-. http://ojs.uv.es/index/php/LEEME/index ISSN: 1575-9563. Editores: Universidad de Valencia y Jesús Tejada. Visibilidad de esta revista: SCOPUS, Emerging Sources Citation Index (Clarivate), EBSCO, CINDOC (CSIC), Citefactor, COPAC, Dialnet, DICE (CSIC), DOAJ, e-revistas (CSIC), EBSCO Premier, ERIH+, Gale Cengage Learning, IN-RECS, IRESIE, LATINDEX, MIAR, OCLC Worldcat, RESH, REDIB, RILM Core Journals, SUDOC, ULRICHS. Esta revista es de acceso libre mediante licencia Creative Commons 4.0 CC by. Política de archivado: etiqueta verde SHERPA-ROMEO. 


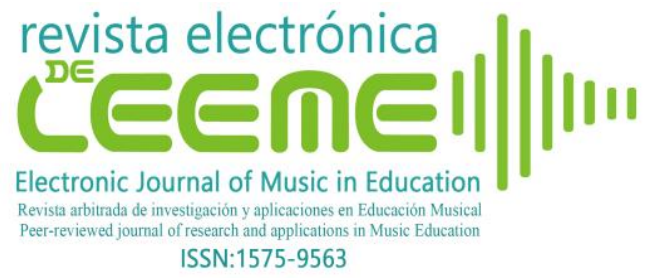

La TAR centra su atención en los procesos de cambio permanente de los actores-red. Latour (2013) señala que cuando un actor circula entre los nodos de una red siempre existe un cambio de lo que está viajando, a este cambio se le denomina traducción; mientras que, cuando los nodos se interconectan a otras redes se trata de una adscripción. Cualquiera de los casos conduce a configurar y reconfigurar la red hasta su estabilización frágil y temporal mediante la inscripción de otros actores en nuevas asociaciones (Callon, Lawand y Rip, 1998/1986). La red, en este sentido, es un proceso de configuración, estabilización o desvanecimiento que no preexiste, sino que se configura en tiempo real a través de controversias.

A partir de la TAR, el estudio de los programas no formales de educación a través de las artes constituye un reto, debido, por un lado, a los orientadores ontológicos, epistemológicos y metodológicos de sus planteamientos no convencionales, a las nociones de agencia no humana y configuración de redes, así como también, por sus métodos afines: etnometodología y etnografía. Mientras que, por el otro, si bien se reconoce que tiene una amplia producción escrita en ciencia, sociología, tecnología, feminismo, geografía cultural, organización y gestión, planificación ambiental, atención de la salud $\mathrm{y}$, ahora, en educación, se trata de un posicionamiento ambiguo, inestable y transgresor de enfoques metodológicos fijos.

En este sentido, el programa no formal de educación a través de las artes es visto como una red donde ocurren asociaciones que la configuran, estabilizan o la desvanecen. Un ejemplo puede ilustrar su configuración: si un joven (Actante) desea inscribirse (Agencia) al programa para aprender a tocar un instrumento se generan desacuerdos (Controversias) entre las estrategias implícitas o explicitas utilizadas (Interesamientos) en la familia y/o el programa (Centros de traducción) para mantener su estabilidad (Traducción). La asociación entre la familia y el programa producen vínculos (Adscripciones) que transforman su relación (Movilización de la red) (Callon, 1986; Latour, 2008, 2013).

Las categorías anteriores, aplicadas al programa educativo no formal a través de las artes, permiten reconocer las tensiones que lo configuran en red (controversias) y, con ello, los interesamientos/motivaciones que generan su estabilización o su desvanecimiento. De tal suerte que podemos dar cuenta de que por el programa educativo circulan valores, intereses, beneficios materiales e inmateriales, actantes, enseñanza y aprendizaje, vínculos con otras redes y, por supuesto, música. Lo anterior, propicia una comprensión más amplia del programa dando cuenta de que solo es posible a través de sus relaciones con otras redes. En un sentido práctico, el estudio de los programas educativos no formales a través de la música permite dar cuenta de su organización y funcionamiento como red, de las relaciones entre aprendizaje de la música con la familia, la educación formal y los lazos entre amigos como formas de asociación o reasociación.

@Elsa Natali González González y Leonel De Gunther Delgado. The content of this article is the sole responsibility of the authors. The Revista Electrónica de LEEME and Universitat de València are not liable for any legal actions that may arise involving the article's content. Revista Electrónica de LEEME - Lista Electrónica Europea de Música en la Educación-. http://ojs.uv.es/index/php/LEEME/index ISSN: 1575-9563. Editores: Universidad de Valencia y Jesús Tejada. Visibilidad de esta revista: SCOPUS, Emerging Sources Citation Index (Clarivate), EBSCO, CINDOC (CSIC), Citefactor, COPAC, Dialnet, DICE (CSIC), DOAJ, e-revistas (CSIC), EBSCO Premier, ERIH+, Gale Cengage Learning, IN-RECS, IRESIE, LATINDEX, MIAR, OCLC Worldcat, RESH, REDIB, RILM Core Journals, SUDOC, ULRICHS. Esta revista es de acceso libre mediante licencia Creative Commons 4.0 CC by. Política de archivado: etiqueta verde SHERPA-ROMEO. 


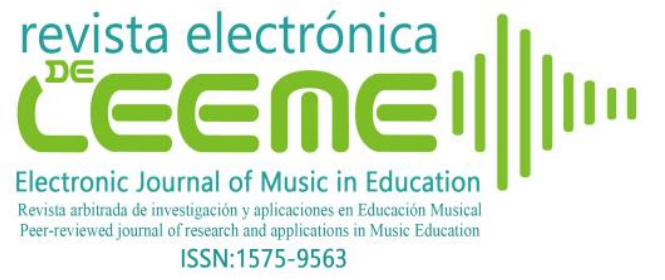

\section{Estrategia metodológica}

Se parte del caso de un programa artístico-educativo no formal establecido por Hermosiarte, el cual es una asociación dedicada a la enseñanza de la música en zonas de vulnerabilidad socioeconómica, ubicada en el estado de Sonora, México. Desde el año 2012 ofrecer alternativas formativas a jóvenes que viven en esos contextos. El modelo de enseñanza replica las modalidades venezolanas y colombianas de enseñanza a través de la música, el cual no forma músicos, sino que utiliza la música como medio formativo, susceptible de encauzar la vida de la juventud y coadyuvar en la restitución del tejido social. Tales modelos fueron empleados durante los levantamientos armados, la lucha contra el narcotráfico y las guerrillas en aquellos países durante los años ochenta y constituyen a la fecha una alterativa para alejar a los jóvenes de ello.

Hermosiarte atiende a 100 jóvenes por año -parte de la población es flotante por lo que la cifra fluctúa-, aproximadamente $29 \%$ son hombres y $71 \%$ mujeres de entre 9 y 17 años. Subsiste a través de donaciones de particulares sea en tiempo de colaboración, especie o dinero y de la búsqueda de financiamiento estatal a través de convocatorias. Las donaciones en tiempo y especie provienen de la colaboración de los miembros de la comunidad, de instituciones gubernamentales, de interesados en el trabajo comunitario, del profesorado de música y, recientemente, de universidades extranjeras y locales que han centrado su interés en cuestiones relativas a la comunicación entre grupos vulnerables o que pueden aportar bienes materiales. Sus horarios de trabajo se organizan durante los domingos y ocasionalmente entre semana, dependiendo de las posibles actuaciones musicales. Durante el 2019, ha sido galardonada como organización filantrópica obteniendo el premio Kybernus al valor ciudadano y ha recibido distinciones por los grupos musicales que ha ido formando, destacando la Orquesta de Cámara Juvenil por el reconocimiento y adscripción como grupo artístico ante el Instituto Sonorense de Cultura ${ }^{2}$.

Esta breve descripción del caso muestra la complejidad de objetos que entran en juego para su configuración: programa educativo no formal, instituciones, personas, comunidad, iniciativa privada, gobierno, universidades, grupos musicales, tiempo, donaciones, política, gestión, etc. En palabras de Hennion (2017), se trata de múltiples objetos como si fueran "resultados provisorios de un tejido heterogéneo de relaciones sin cesar experimentadas, testeadas, reorganizadas, para producir otros objetos, sin que se pueda distinguir de manera estable soporte y contenido, red y actores, productos y usuarios..." (p.4).

Para el desarrollo metodológico, empleamos recursos de la etnografía, entendida como "[...] tratar de leer (en el sentido de interpretar un texto) un manuscrito extranjero, borroso,

Véase en: http://aztecasonora.com/2019/05/gana-hermosiarte-premio-al-valor-ciudadano/ y en http://isc.gob.mx/devel/que-hacemos/artes/musica/orquesta-de-camara-juvenil/

@Elsa Natali González González y Leonel De Gunther Delgado. The content of this article is the sole responsibility of the authors. The Revista Electrónica de LEEME and Universitat de València are not liable for any legal actions that may arise involving the article's content. Revista Electrónica de LEEME - Lista Electrónica Europea de Música en la Educación-. http://ojs.uv.es/index/php/LEEME/index ISSN: 1575-9563. Editores: Universidad de Valencia y Jesús Tejada. Visibilidad de esta revista: SCOPUS, Emerging Sources Citation Index (Clarivate), EBSCO, CINDOC (CSIC), Citefactor, COPAC, Dialnet, DICE (CSIC), DOAJ, e-revistas (CSIC), EBSCO Premier, ERIH+, Gale Cengage Learning, IN-RECS, IRESIE, LATINDEX, MIAR, OCLC Worldcat, RESH, REDIB, RILM Core Journals, SUDOC, ULRICHS. Esta revista es de acceso libre mediante licencia Creative Commons 4.0 CC by. Política de archivado: etiqueta verde SHERPA-ROMEO. 
plagado de elipsis, de incoherencias, de sospechosas enmiendas y de comentarios tendenciosos y además escrito, no en las grafías convencionales de representación sonora, sino en ejemplos volátiles de conducta modelada" (Geertz, 2003, p.24).

En este sentido, se utilizó la técnica de observación participante durante 18 meses, para registrar in situ el funcionamiento y organización de HermosiArte. Además, se realizaron 12 entrevistas semiestructuradas al grupo de violín avanzado, el cual destaca por su trayectoria, reconocimiento social y consolidación. Está formado por mujeres de entre 12 y 17 años, además se encuentran en los niveles "D" y "E" considerados los niveles más bajos de bienestar, (Índice de Nivel Socioeconómico AMAI, 2018) ${ }^{3}$. Tal entrevista consistió en 28 ítems, distribuidos en 6 secciones: datos generales, del programada educativo no formal, de la familia, de la escuela formal, de las relaciones entre amigos y, finalmente, una sección de contexto socioeconómico relacionado con AMAI. Los registros y las entrevistas recuperan información relativa a las categorías: centros de traducción, controversias, interesamientos, actantes, traducciones, adscripciones y movimientos de la red: configuración, estabilización y desvanecimiento. Además, se solicitó consentimiento informado a los padres y, se guardó la confidencialidad de los datos obtenidos.

El procedimiento de análisis consistió en poner en relación las diferentes categorías señaladas en el párrafo anterior y los datos obtenidos. En este sentido, seguimos a los actores, lo que saben, lo que hacen, y el cómo y por qué lo hacen (Latour, 1999). La atención se focalizó en dos centros de traducción: el programa de Hermosiarte y la red de lazos entre amigos. Dentro de estos se recuperaron las controversias como traducción de las informaciones, así como los actantes (qué o quién hace hacer a otros). Igualmente se focalizó la atención en los interesamientos de cada centro de traducción y los intereses propios de los actantes. Se recuperan los vínculos a través de la adscripción (mediaciones que entrelazan a los centros de traducción), para reconocer su asociación, y, en conjunto los procesos de configuración, estabilización o desvanecimiento de la red, obteniendo nuevas respuestas sobre lo que hace la educación a través de las artes en los grupos humanos.

\subsection{Ensamblando la red de Hermosiarte. Seguir las asociaciones}

La red de Hermosiarte da cuenta de cómo una red ensambla lo social y cómo se inscribe en otras redes (como centros de traducción), de cómo se estabiliza y se desestabiliza y, a la vez, guía el curso de su acción como programa no formal de educación a través de las artes.

\footnotetext{
${ }^{3}$ El Nivel Socioeconómica (NSE) de la Asociación Mexicana de Agencias de Inteligencia de Mercado y Opinión (AMAI) es una regla basada en un modelo estadístico que permite clasificar los hogares en 7 niveles de bienestar basada en un marco conceptual que agrupa seis dimensiones: capital humano, infraestructura práctica, conectividad y entretenimiento, infraestructura sanitaria, planeación y futuro e infraestructura básica y espacio (véase en: Asociación Mexicana de Agencias de Inteligencia de Mercado y Opinión [AMAI]. (2018). Índice de Niveles Socioeconómicos. Regla 6x7. Recuperado de: https://nse.amai.org/nse/
}

@Elsa Natali González González y Leonel De Gunther Delgado. The content of this article is the sole responsibility of the authors. The Revista Electrónica de LEEME and Universitat de València are not liable for any legal actions that may arise involving the article's content. Revista Electrónica de LEEME - Lista Electrónica Europea de Música en la Educación-. http://ojs.uv.es/index/php/LEEME/index ISSN: 1575-9563. Editores: Universidad de Valencia y Jesús Tejada. Visibilidad de esta revista: SCOPUS, Emerging Sources Citation Index (Clarivate), EBSCO, CINDOC (CSIC), Citefactor, COPAC, Dialnet, DICE (CSIC), DOAJ, e-revistas (CSIC), EBSCO Premier, ERIH+, Gale Cengage Learning, IN-RECS, IRESIE, LATINDEX, MIAR, OCLC Worldcat, RESH, REDIB, RILM Core Journals, SUDOC, ULRICHS. Esta revista es de acceso libre mediante licencia Creative Commons 4.0 CC by. Política de archivado: etiqueta verde SHERPA-ROMEO. 
Además, presenta las controversias que disparan interesamientos entre los actantes y configuran su adscripción a otras redes (centros de traducción). Se trata pues de un flujo complejo que permite reconocer su organización y su potencialidad en una comunidad de vulnerabilidad socioeconómica, a través de una descripción abierta (Latour, 2008). Lo que se ensambla en la red, lo que se estabiliza o se desvanece en ella y lo que produce son resultado de las controversias, interesamientos y adscripciones puestos en movimiento en cada una de las actividades cotidianas (Fenwick y Edwards, 2012). En este sentido, investigar significa crear interesamientos, colocarnos entre los estudios previos sobre los programas no formales de educación a través de las artes y la tendencia esquiva de la ciencia tradicional para valorarlos.

Crear interesamientos significa reconocer en el mapeo de las controversias: familia, escuela, amigos, programa, etc.; la posibilidad de reensamblar lo social, de relacionar sin unir, de mantener la contabilidad lógica del dominio al que pertenecen los objetos que se han mezclado. De ahí que otras preguntas se vuelvan urgentes: ¿qué mundo están ensamblando las redes?, ¿cómo se ensamblan y a través de qué centros de traducción y de qué actantes?, ¿cómo se estabilizan para perdurar?, ¿qué dominios de sentido comparten para crear comunidad? (Latour, 2010; 2013). En función de los datos obtenidos, damos cuenta de ello en la figura 1.

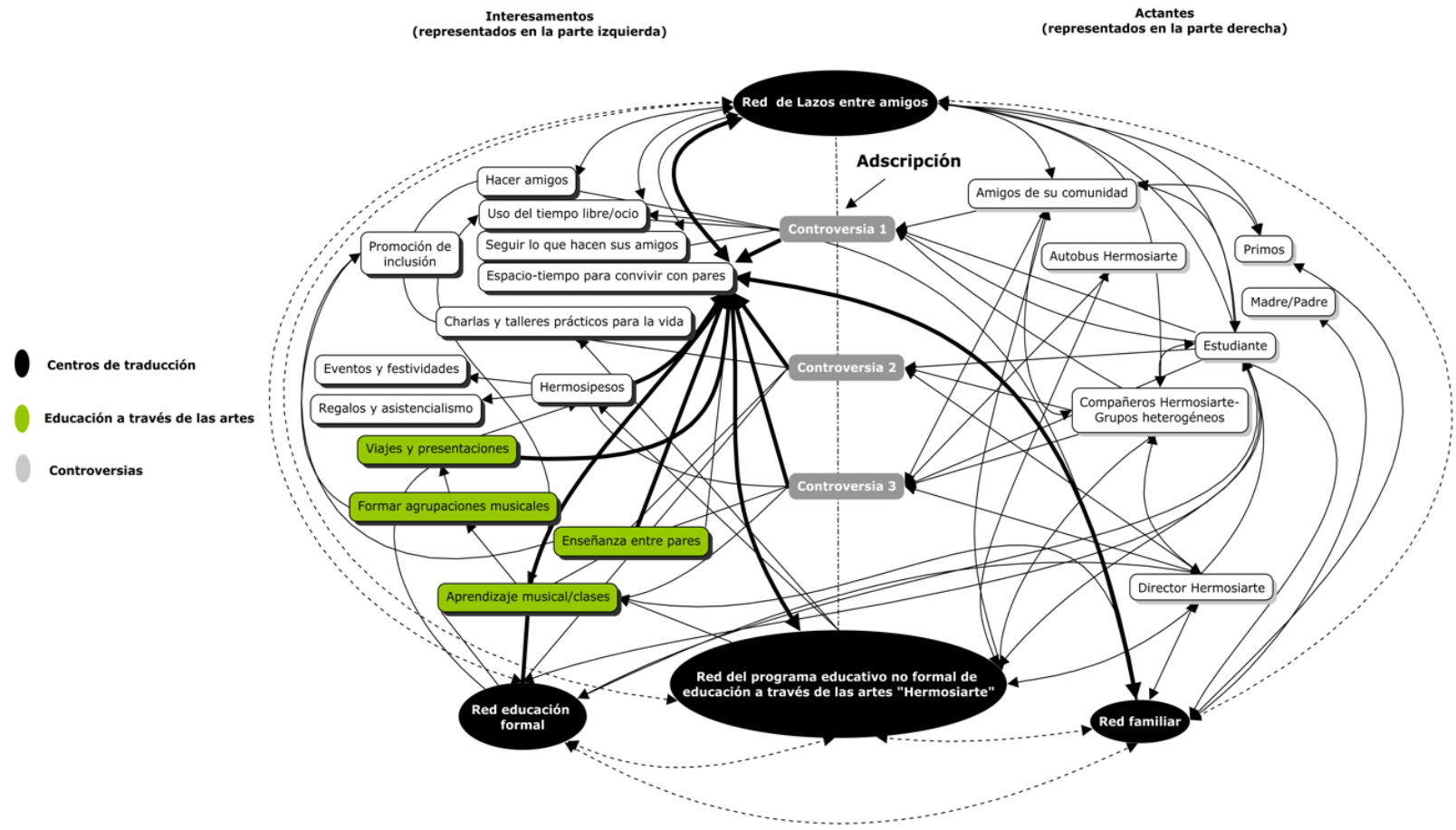

Figura 1. Ensamblaje de la red de relaciones de Hermosiarte. Fuente: elaboración propia

@Elsa Natali González González y Leonel De Gunther Delgado. The content of this article is the sole responsibility of the authors. The Revista Electrónica de LEEME and Universitat de València are not liable for any legal actions that may arise involving the article's content. Revista Electrónica de LEEME - Lista Electrónica Europea de Música en la Educación-. http://ojs.uv.es/index/php/LEEME/index ISSN: 1575-9563. Editores: Universidad de Valencia y Jesús Tejada. Visibilidad de esta revista: SCOPUS, Emerging Sources Citation Index (Clarivate), EBSCO, CINDOC (CSIC), Citefactor, COPAC, Dialnet, DICE (CSIC), DOAJ, e-revistas (CSIC), EBSCO Premier, ERIH+, Gale Cengage Learning, IN-RECS, IRESIE, LATINDEX, MIAR, OCLC Worldcat, RESH, REDIB, RILM Core Journals, SUDOC, ULRICHS. Esta revista es de acceso libre mediante licencia Creative Commons 4.0 CC by. Política de archivado: etiqueta verde SHERPA-ROMEO. 
La Figura 1 presenta y organiza una parte de la configuración de la red. En los extremos superior e inferior se localizan los centros de traducción o redes (en negro) conformados por el programa no formal de educación a través de las artes, los lazos entre amigos, la familia y la educación formal. En el centro, las controversias (en gris), como disparadores de la adscripción entre el programa y los lazos entre amigos. En el lado derecho, los actantes y, en el izquierdo, los interesamientos. Al lado inferior izquierdo, las principales prácticas musicales de la educación a través de las artes (en verde). Las líneas de mayor grosor resaltan la densidad de las conexiones entre los nodos, destacando su convergencia en el interesamiento de "espaciotiempo para convivir con las pares". En los apartados siguientes se ejemplifica el análisis del ensamblaje de la red.

\subsection{Centros de traducción: Hermosiarte y lazos entre amigos}

Hermosiarte promueve la creación de lazos entre amigos al asociarlos y establecer vínculos entre ellos, donde las controversias generan y producen nuevas adscripciones, las cuales configuran, estabilizan o desvanecen la red. Tal es el caso de la creación de la rondalla y el tiempo que pasan juntos sus integrantes entre clases, ensayos, presentaciones y espacios de convivencia (espacio-tiempo para convivir con pares, figura 1). Contemos una historia la cual asocia dos grupos de Hermosiarte: el de rondalla y el de violín avanzado; que presenta la controversia 1. Durante el año 2019, hubo un evento que sirve de preámbulo para colocar nuestra mirada en el centro de traducción de los lazos entre amigos. El grupo de la rondalla integrado por cinco estudiantes establecieron un lazo de amistad al estar juntos por más de ocho meses, sin embargo, abandonaron el programa. El director y fundador de Hermosiarte lo comprende así:

una estudiante -del grupo de violín avanzado- se puso de novia con uno de los chicos de la rondalla que abandonó el programa. Poco después terminaron. Así que para no hacerse conflicto decidieron no verse y el chico dejó de venir al programa y, también, sus amigos. Estos jovencitos eran los que mejor tocaban.

En una entrevista posterior con la ahora exnovia pudimos corroborar la historia. Ella agrega, "dejaron de venir porque ya no estarían juntos (los amigos)" (E04). Es decir, se siguen unos a otros aun cuando su afinidad y vocación por la música siga presente. Este breve relato introductorio muestra que la creación de interesamientos/motivaciones de los jóvenes para asistir al programa es compartir tiempo con sus amigos, que las prácticas instrumentales en ensayos, clases y presentaciones lograron establecer lazos duraderos. Así que, al no estar juntos, su asistencia carece de motivaciones y deciden retirarse. Este ejemplo muestra el movimiento de los actantes, de lo que sucede cuando están conectados con otras redes en movimiento. El grupo de rondalla se desvanece, a pesar de que era el segundo de mayor participación en eventos y

@Elsa Natali González González y Leonel De Gunther Delgado. The content of this article is the sole responsibility of the authors. The Revista Electrónica de LEEME and Universitat de València are not liable for any legal actions that may arise involving the article's content. Revista Electrónica de LEEME - Lista Electrónica Europea de Música en la Educación-. http://ojs.uv.es/index/php/LEEME/index ISSN: 1575-9563. Editores: Universidad de Valencia y Jesús Tejada. Visibilidad de esta revista: SCOPUS, Emerging Sources Citation Index (Clarivate), EBSCO, CINDOC (CSIC), Citefactor, COPAC, Dialnet, DICE (CSIC), DOAJ, e-revistas (CSIC), EBSCO Premier, ERIH+, Gale Cengage Learning, IN-RECS, IRESIE, LATINDEX, MIAR, OCLC Worldcat, RESH, REDIB, RILM Core Journals, SUDOC, ULRICHS. Esta revista es de acceso libre mediante licencia Creative Commons 4.0 CC by. Política de archivado: etiqueta verde SHERPA-ROMEO. 
conciertos musicales. En términos de Latour (2008), muestra la red en movimiento: su desvanecimiento.

Los siguientes ejemplos (abajo) muestran la complejidad de las redes y la estabilización de los lazos entre amigos, surgen en los espacios y tiempos del aprendizaje musical, de la enseñanza entre pares, de la formación de agrupaciones musicales y de los viajes y presentaciones. Son narrativas presentes en la memoria de los actantes; otras son acontecimientos performativos, los cuales pueden o no continuar. De ahí lo precario de la red, pero, a la vez, su capacidad de ensamblarse. Se deja entrever en ellas las temporalidades de los actantes y la construcción social del tiempo en su configuración. A continuación, se ilustran las controversias 2 y 3 .

El ejemplo 1 (abajo) representa una configuración de la red entre el programa, los lazos entre amigos y la educación formal, mientras que en los ejemplos del 2 al 5 muestran la configuración entre el programa, los lazos entre amigos y familia. Se trata de la inscripción de múltiples redes superpuestas, de inscripciones que expanden la red, de asociaciones entre redes, donde lo social es un ensamblaje incesante como lo expresa Latour (2010, 2013), manifestando la complejidad en la estabilización de la red en una compleja trama de traducciones y adscripciones:

(1) En el programa nos enseñaron a hacer minicuentos (realizados entre pares) y comencé a escribir y he logrado muchas cosas con ello, hace poco se lo mostré a mi maestro de literatura de la prepa (educación formal) y me dijo que mi cuento va a aparecer en la revista de la escuela y me sentí muy bien (E01).

(2) Iba caminando con dos de mis amigas en la calle y el director de Hermosiarte nos encontró y nos preguntó si queríamos aprender a tocar violín y le dijimos que sí y nos apuntó y nos dijo la fecha para empezar las clases y ese día fui con mis amigas cerca de un parque en unas oficinas que le prestaron para las clases (E08).

(3) Mis mejores amigas son las de aquí -las del programa-, con ellas me las llevo a todos lados. Nos vamos al cine, o vamos una plaza, al mall, o andamos juntas a veces en pijamadas, o nos juntamos nomas en una casa a practicar -el instrumento- y hacemos comida juntas o así $(\mathrm{E} 02)^{4}$.

(4) Ahora salgo más con mis amigos de aquí -del programa-, salimos a muchas partes, me divierto mucho con ellos...más porque nos sacan -del programa- a otros lugares juntos principalmente parques de recreación y viajes de las presentaciones- (E03).

(5) Siempre me gusta venir porque en mi casa no hago nada y aquí me entretengo mucho y aparte hago amigos, hago algo útil -aprender a tocar un instrumento- (E04).

La estabilización de Hermosiarte como una red o su ensamblaje con otras consiste en la composición de un mundo (Latour, 2010; 2013), cuya posibilidad surge de la agencia de los actantes, en la cotidianidad de los actos a veces desapercibidos que, como pequeños vínculos en una red, puede conducir al cambio y producir una comunidad con un sentido compartido a través de sus asociaciones.

\footnotetext{
${ }^{4}$ Se utiliza la nomenclatura "E02" para referirse al estudiante con asignación numérica '02' por concepto de anonimato. En lo sucesivo el número varía según el informante como nombre clave.
}

@Elsa Natali González González y Leonel De Gunther Delgado. The content of this article is the sole responsibility of the authors. The Revista Electrónica de LEEME and Universitat de València are not liable for any legal actions that may arise involving the article's content. Revista Electrónica de LEEME - Lista Electrónica Europea de Música en la Educación-. http://ojs.uv.es/index/php/LEEME/index ISSN: 1575-9563. Editores: Universidad de Valencia y Jesús Tejada. Visibilidad de esta revista: SCOPUS, Emerging Sources Citation Index (Clarivate), EBSCO, CINDOC (CSIC), Citefactor, COPAC, Dialnet, DICE (CSIC), DOAJ, e-revistas (CSIC), EBSCO Premier, ERIH+, Gale Cengage Learning, IN-RECS, IRESIE, LATINDEX, MIAR, OCLC Worldcat, RESH, REDIB, RILM Core Journals, SUDOC, ULRICHS. Esta revista es de acceso libre mediante licencia Creative Commons 4.0 CC by. Política de archivado: etiqueta verde SHERPA-ROMEO. 


\section{Discusión y conclusiones}

En los apartados anteriores construimos primero el problema de investigación planteando que la educación a través de las artes presenta beneficios al desarrollo humano, pero se desconoce el proceso que sigue para alcanzarlos, de ahí que nos preguntemos, ¿cómo se configura la red de Hermosiarte, en particular entre el programa y los lazos entre amigos? Y, con ello, ¿cuáles son las implicaciones de la educación a través de las artes? Segundo, presentamos los principales elementos de la TAR para abordar el problema, tercero, se describió la metodología basada en la etnografía para la recolecta y análisis de los datos y finalmente se dan a conocer algunos de los resultados centrados en dos redes: el programa y los lazos entre amigos. En este apartado, discutimos los resultados y las principales conclusiones.

La red de Hemosiarte se configura a partir de las controversias, las cuales funcionan como disparadores entre los actantes y los interesamientos, produciendo adscripciones hacia otras redes, en particular hacia los lazos entre amigos. Así, todo centro de traducción produce controversias que potencian los pequeños vínculos para mantener ensamblada la red (véase ejemplos 1-5, arriba). Lo social es sin duda una construcción. Las redes se construyen y no preexisten como entidades anteriores. Como señala Latour (2008), es el resultado de seguir las asociaciones para dar cuenta de su construcción.

La agencia o intencionalidad de los actantes que operan la red se traduce en el fortalecimiento de los lazos que unen a los estudiantes y por ende en la creación y mantenimiento del programa. La TAR es útil para comprender lo que hace el arte en programas educativos no formales, dado que es posible cartografiar el proceso que ensambla las practicas cotidianas como la enseñanza y el aprendizaje de la música así como las distintas actividades que lo hacen posible como las charlas y talleres prácticos para la vida (apoyo a la educación formal), el tiempo libre entre las actividades para la convivencia entre pares, eventos y festividades, salidas a centros recreativos, regalos y asistencialismos (apoyo a la familia), entre otros.

La TAR, permite, además, reconocer que aun cuando la red de Hermosiarte se caracteriza por ser una red inestable, precaria en una zona también inestable y precaria, en cuanto a su estabilización y materialización, es una alternativa viable para comprender su valía frente a un sistema de valoración que, si bien no escatima en reconocer el valor general de la misma, cuestiona su valor como medio para potenciar el desarrollo humano. La Figura 2, abajo, sinteriza estas ideas:

@Elsa Natali González González y Leonel De Gunther Delgado. The content of this article is the sole responsibility of the authors. The Revista Electrónica de LEEME and Universitat de València are not liable for any legal actions that may arise involving the article's content. Revista Electrónica de LEEME - Lista Electrónica Europea de Música en la Educación-. http://ojs.uv.es/index/php/LEEME/index ISSN: 1575-9563. Editores: Universidad de Valencia y Jesús Tejada. Visibilidad de esta revista: SCOPUS, Emerging Sources Citation Index (Clarivate), EBSCO, CINDOC (CSIC), Citefactor, COPAC, Dialnet, DICE (CSIC), DOAJ, e-revistas (CSIC), EBSCO Premier, ERIH+, Gale Cengage Learning, IN-RECS, IRESIE, LATINDEX, MIAR, OCLC Worldcat, RESH, REDIB, RILM Core Journals, SUDOC, ULRICHS. Esta revista es de acceso libre mediante licencia Creative Commons 4.0 CC by. Política de archivado: etiqueta verde SHERPA-ROMEO. 


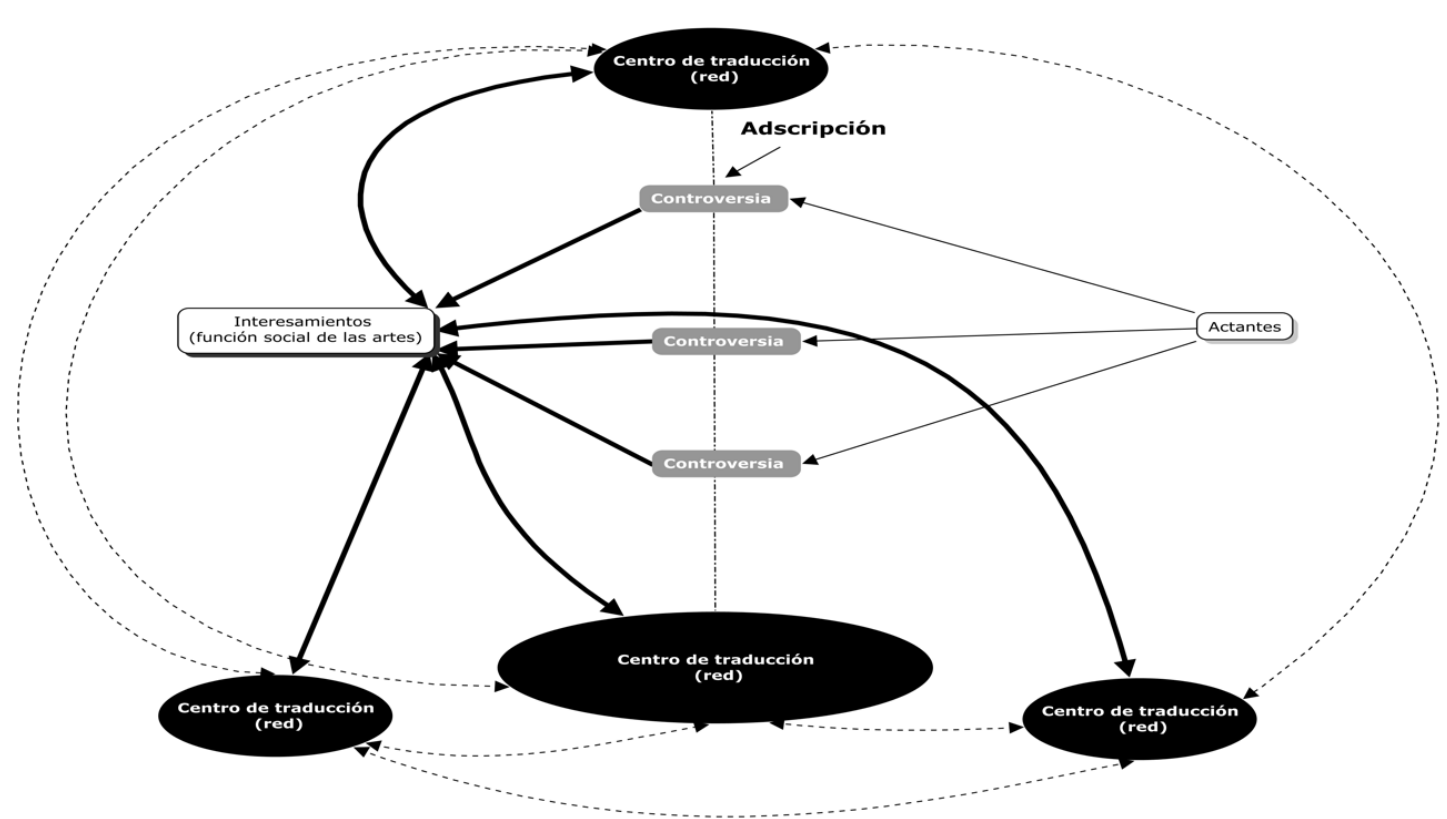

Figura 2: Modelo teórico-empírico. Teoría del actor-red (ANT). Fuente: elaboración propia

La configuración en red de Hermosiarte también permite observar varias implicaciones derivadas de las asociaciones que produce:

1. Promueve diferentes experiencias educativas y de vida a través de las artes y no sólo del aprendizaje de la música.

2. Genera asociaciones que suscitan el compromiso y la colaboración entre los actantes.

3. La configuración da cuenta del proceso de construcción de la red a partir de las pequeñas partes que la constituyen.

4. Permite reconocer el proceso que está detrás de las aportaciones o cambios que le son atribuidos a la educación a través de las artes en la construcción de un mundo posible.

5. Favorece dinámicas de integración entre los distintos centros de traducción donde se desenvuelven los estudiantes, brindándoles otras opciones de vida que les permiten reconstruir nuevas representaciones del mundo en que viven.

De lo anterior, se desprende la idea de que la red de Hermosiarte se moviliza traduciendo informaciones de diferentes redes o centros de traducción (lazos entre amigos, familia, educación formal) para configurar la función social de la educación a través de las artes.

@Elsa Natali González González y Leonel De Gunther Delgado. The content of this article is the sole responsibility of the authors. The Revista Electrónica de LEEME and Universitat de València are not liable for any legal actions that may arise involving the article's content. Revista Electrónica de LEEME - Lista Electrónica Europea de Música en la Educación-. http://ojs.uv.es/index/php/LEEME/index ISSN: 1575-9563. Editores: Universidad de Valencia y Jesús Tejada. Visibilidad de esta revista: SCOPUS, Emerging Sources Citation Index (Clarivate), EBSCO, CINDOC (CSIC), Citefactor, COPAC, Dialnet, DICE (CSIC), DOAJ, e-revistas (CSIC), EBSCO Premier, ERIH+, Gale Cengage Learning, IN-RECS, IRESIE, LATINDEX, MIAR, OCLC Worldcat, RESH, REDIB, RILM Core Journals, SUDOC, ULRICHS. Esta revista es de acceso libre mediante licencia Creative Commons 4.0 CC by. Política de archivado: etiqueta verde SHERPA-ROMEO. 


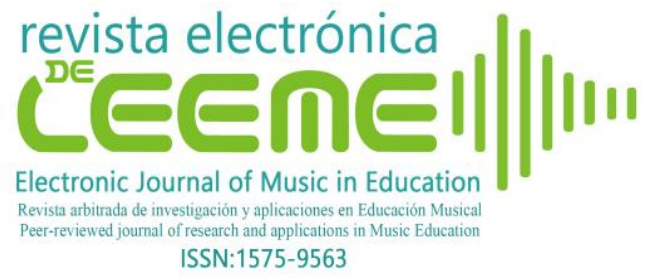

Es decir, el programa como acontecimiento no discreto extiende su flujo por otras redes que entrelazan la de los propios estudiantes con otras, logrando un proceso que integra su mundo conocido y por conocer, ampliando sus perspectivas y formas de ver y hacer en el mundo, produciendo intencionalmente en ellos desarrollo humano. En términos sociotécnicos cambia lo que vehicula los contenidos de las otras redes. Por las redes de los programas de educación a través de la música transita más que sólo música.

Si bien es cierto el uso de la TAR permite llegar a las consideraciones anteriores dados sus alcances, también es cierto que presenta limitaciones, entre las que destacamos que sólo permite describir la composición de la red, de las conexiones que ocurren en tiempo real y de sus consecuencias, resultado problemático considerar el principio de agencia que involucra a sujetos y objetos como actantes intencionales en la configuración de la red (Larrión, 2019). Aun así, la TAR ofrece una forma de pensar relacionalmente. Solo en la relación hay sentido, otra forma de concebir lo social y mostrar el proceso de ensamblaje de lo que hacen los programas en red.

Nos parece claro que la investigación de los programas no formales de educación a través de las artes requiere un tipo de estudio que nos los atomice; sino que los ponga en relación con el conjunto de redes con los que se asocian. Decimos lo anterior, porque la existencia del programa Hermosiarte depende de sus relaciones en red. De aquí que sea necesario continuar la investigación atrayendo y adecuando otros posicionamientos teóricos para fortalecer las limitaciones propias de la TAR.

\section{Referencias}

Ángel-Alvarado, R y Lira-Cerda, J. (2017). Instalaciones y recursos educativos para la educación musical según la representación social de los estudiantes chilenos. Revista electrónica de LEEME, 40, 19-31. doi:10.7203/LEEME.40.10412

Berrón, E. (2017). Integración creativa de la música Pop-Rock en el aula. Revista Electrónica de LEEME, 39, 1-20. doi:10.7203/LEEME.39.10070

Callon, M., Lawand, J. y Rip, A. (1998/1986). Mapping the dynamics of science and technology: sociology of science in the real world. Basingstoke: Macmillan Press.

Christophersen, C. (2015). Changes and challenges in music education: reflections on a Norwegian arts-in-education program, Music Education Research, 17(4), 365-380, doi:10.1080/14613808.2014.930119

Consejo Nacional de Evaluación de la Política de Desarrollo Social [CONEVAL] y Fondo Internacional de Emergencia de las Naciones Unidas para la Infancia [UNICEF]. (2015).

@Elsa Natali González González y Leonel De Gunther Delgado. The content of this article is the sole responsibility of the authors. The Revista Electrónica de LEEME and Universitat de València are not liable for any legal actions that may arise involving the article's content. Revista Electrónica de LEEME - Lista Electrónica Europea de Música en la Educación-. http://ojs.uv.es/index/php/LEEME/index ISSN: 1575-9563. Editores: Universidad de Valencia y Jesús Tejada. Visibilidad de esta revista: SCOPUS, Emerging Sources Citation Index (Clarivate), EBSCO, CINDOC (CSIC), Citefactor, COPAC, Dialnet, DICE (CSIC), DOAJ, e-revistas (CSIC), EBSCO Premier, ERIH+, Gale Cengage Learning, IN-RECS, IRESIE, LATINDEX, MIAR, OCLC Worldcat, RESH, REDIB, RILM Core Journals, SUDOC, ULRICHS. Esta revista es de acceso libre mediante licencia Creative Commons 4.0 CC by. Política de archivado: etiqueta verde SHERPA-ROMEO. 
Pobreza y derechos sociales de niñas, niños y adolescentes en México, 2014. Recuperado de: https://www.unicef.org/mexico/spanish/MX Pobreza derechos.pdf

Fenwick, T.J.A. y Edwards, R. (2010). Actor-network theory in education. New York: Routledge Taylor y Francis Group.

Fenwick, T. y Edwards, R. (2012). Researching Education Through Actor-Network Theory. Sussex, Reino Unido: Wiley-Blackwell.

Geertz, C. (1973). La interpretación de las culturas. Barcelona, España: Gedisa.

Hennion, A. (2017). De una sociología de la mediación a una pragmática de las vinculaciones. Retrospectiva de un recorrido sociológico dentro del CSI. Cuestiones de sociología, 16, e32. Recuperado de: http://memoria.fahce.unlp.edu.ar/art revistas/pr.8194/pr8194.pdf

Larrión, J. (2019). Actor-network theory. Synthesis and evaluation of Bruno Latour's post-social drift. Revista Española de Sociología, 28(2), 323-341. doi:10.22325/fes/res.2019.0

Latour, B. (1999). On recalling ANT. En J. Law y J. Hassard (Eds.), Actor network theory and after (pp.15-25). Oxford: Blackwell Publishers: The Sociological Review.

Latour, B. (2008). Reensamblar lo social. Una introducción a la teoría del actor-red. Buenos Aires, Manantial.

Latour, B. (2010). Cogitamus Seis cartas sobre las humanidades científicas. Barcelona: Paidós Editores.

Latour, B. (2013). Investigación sobre los modos de existencia. Una antropología de los modernos. Buenos Aires: Paidós.

Machover, T. (2007). My Cello. En S. Turkle (Ed.), Evocative objects: things we think with (pp.14-21). Cambridge: The MIT Press.

Morin, E. (2002). La cabeza bien puesta. Repensar la reforma. Reformar el pensamiento. Bases para una reforma educativa. Buenos Aires: Nueva visión.

Piaget, J. (1975). Problemas de psicología genética. Barcelona: Ariel.

Rusinek, G. (2008). Disaffected learners and school musical culture: an opportunity for inclusion. Research Studies in Music Education, 30(1), 9-23. doi:10.1177/1321103x08089887

@Elsa Natali González González y Leonel De Gunther Delgado. The content of this article is the sole responsibility of the authors. The Revista Electrónica de LEEME and Universitat de València are not liable for any legal actions that may arise involving the article's content. Revista Electrónica de LEEME - Lista Electrónica Europea de Música en la Educación-. http://ojs.uv.es/index/php/LEEME/index ISSN: 1575-9563. Editores: Universidad de Valencia y Jesús Tejada. Visibilidad de esta revista: SCOPUS, Emerging Sources Citation Index (Clarivate), EBSCO, CINDOC (CSIC), Citefactor, COPAC, Dialnet, DICE (CSIC), DOAJ, e-revistas (CSIC), EBSCO Premier, ERIH+, Gale Cengage Learning, IN-RECS, IRESIE, LATINDEX, MIAR, OCLC Worldcat, RESH, REDIB, RILM Core Journals, SUDOC, ULRICHS. Esta revista es de acceso libre mediante licencia Creative Commons 4.0 CC by. Política de archivado: etiqueta verde SHERPA-ROMEO. 
Silva, C. (2011). La teoría del actor-red tal como yo la imagino. Breve ensayo sobre el cosmos semáfórico. Athenea digital, 11(1), 203-215. Recuperado de: https://atheneadigital.net/article/view/v11-n1-silva

Tirado, F. y Domènech, M. (2005). Asociaciones heterogéneas y actantes: El giro postsocial de la teoría del actor-red. AIBR Revista de Antropología Iberoamericana, número especial, 1-26. Recuperado de: http://www.redalyc.org/pdf/623/62309905.pdf

Turkle, S. (Ed.) (2007). Evocative objects: things we think with. Cambridge: The MIT Press.

@Elsa Natali González González y Leonel De Gunther Delgado. The content of this article is the sole responsibility of the authors. The Revista Electrónica de LEEME and Universitat de València are not liable for any legal actions that may arise involving the article's content. Revista Electrónica de LEEME - Lista Electrónica Europea de Música en la Educación-. http://ojs.uv.es/index/php/LEEME/index ISSN: 1575-9563. Editores: Universidad de Valencia y Jesús Tejada. Visibilidad de esta revista: SCOPUS, Emerging Sources Citation Index (Clarivate), EBSCO, CINDOC (CSIC), Citefactor, COPAC, Dialnet, DICE (CSIC), DOAJ, e-revistas (CSIC), EBSCO Premier, ERIH+, Gale Cengage Learning, IN-RECS, IRESIE, LATINDEX, MIAR, OCLC Worldcat, RESH, REDIB, RILM Core Journals, SUDOC, ULRICHS. Esta revista es de acceso libre mediante licencia Creative Commons 4.0 CC by. Política de archivado: etiqueta verde SHERPA-ROMEO. 